\title{
Joint Machine-Type Device Selection and Power Allocation for Buffer-Aided Cognitive M2M Communication
}

\author{
Mostafa Darabi ${ }^{\dagger}$, Behrouz Maham ${ }^{\dagger \ddagger}$, Walid Saad ${ }^{\sharp}$, Abolfazl Mehbodniya ${ }^{\S}$, and Fumiyuki Adachi ${ }^{\S}$ \\ ${ }^{\dagger}$ School of ECE, College of Engineering, University of Tehran, Iran \\ ${ }^{\ddagger}$ Department of Electrical and Electronic Engineering, School of Engineering, Nazarbayev University, Astana, Kazakhstan \\ \#Wireless@VT, Bradley Department of Electrical and Computer Engineering, Blacksburg, VA 24060, USA \\ ${ }^{\S}$ Dept. of Communication Engineering, Graduate School of Engineering, Tohoku University, Sendai, Japan \\ emails: \{mostafa.darabi,bmaham\}@ut.ac.ir,walids@vt.edu, \{mehbod@mobile.ecei, adachi@ecei\}.tohoku.ac.jp
}

\begin{abstract}
In this paper, a cognitive machine-to-machine (M2M) communication network is considered, in which a cellular network shares the spectrum with the M2M communication network with $M$ machine-type devices (MTDs), one half-duplex relay, and one MTD gateway for data gathering. One key challenge is that in the future $5 \mathrm{G}$ wireless networks, there will be billions of those small MTDs, and therefore, a MTD selection protocol is required for managing data transmission between MTDs. A joint buffer-aided MTD selection and power allocation protocol is proposed to maximize the MTDs' sum-rate provided that the induced interference to the cellular network is limited. In particular, in the proposed scheme, at each time slot and each subcarrier, the cognitive M2M network optimally decides on whether to be silent or to select either the relay or one of the MTDs for data transmission. To this end, for each MTD, there exists a buffer at the relay to avoid data loss. The closed-form expressions for the power coefficients of MTDs are calculated. Simulation results show that the proposed policy improves the sum-rate of the CM2M network in comparison with the other proposed schemes for M2M communication without buffer.
\end{abstract}

Index Terms-Buffer-aided cognitive M2M communication, machine-type device selection, power allocation, 5G network.

\section{INTRODUCTION}

Machine-to-machine (M2M) communications have gained significant attention recently and it is anticipated that by 2020, the number of machine-type devices (MTDs) will be in the billions [1]. In M2M communication, wireless MTDs such as sensors, metering devices, and actuators must be interconnected via reliable wireless links. In general, M2M communication networks have short data packets, low data transmission rate, and are in low priority classes. For coping with such M2M characteristics, the idea of capillary networks has recently attracted attention [2]. In such networks, the burden of M2M communication is carried in a hybrid structure in which data of MTDs is not transmitted directly to the cellular network. Instead, MTDs close together exchange information with one another and access the cellular network through gateways or aggregations [3].

In the future $5 \mathrm{G}$ wireless networks, there will be billions of MTDs, and therefore, M2M communications require new spectrum resources. An innovative approach called cognitive

The research results presented in this material have been supported by the U.S. National Science Foundation under Grant CNS-1460333 and by "Towards Energy-Efficient Hyper-Dense Wireless Networks with Trillions of Devices", the Commissioned Research of National Institute of Information and Communications Technology (NICT), JAPAN and KDDI foundation research grant, "Energy-Efficient Radio Resource Management for Next Generation Wireless Network".
M2M (CM2M) has been recently proposed to defeat the lack of the radio spectrum resources. One way to incorporate M2M communication is in a cognitive manner such that the cellular network owns the spectrum and shares it with the MTDs [4]. Applying cognitive methods to M2M communication networks is a promising approach [5]. In particular, in [6], an extensive survey is done to discuss how cognitive radio mechanisms are exploited in M2M environments. In [3], a cognitive and opportunistic relay policy is introduced for CM2M network in order to sense the spectrum used by a cellular network, to cancel the interference, and to apply opportunistic relay selection. In [7], a new medium access control protocol, called data aided cognitive technique, is proposed for the CM2M network in order to improve the sum-rate of the MTDs while the overall delay of system is managed.

The main contribution of this paper is to propose a novel joint buffer-aided MTD selection and power allocation policy for MTDs in a capillary CM2M network. At each time slot, according to the instantaneous or statistical channel state information (CSI) of the involved links, the CM2M network optimally decides to be silent or to select either the relay or one of the MTDs such that the data transmission of the cellular network and MTDs become error-free. For a successful data transmission in the cellular network, the interference of the MTDs at the base station must remain below a predefined threshold. In addition, due to the low data transmission rate of the MTDs and unknown instantaneous CSI of the interfering links (I-CSI-I) from the cellular network to the CM2M network, the induced interference of the user equipments (UEs) to the MTDs is treated as noise. Furthermore, in the proposed power allocation policy, the closed-form expressions for the power coefficients of MTDs are calculated. Simulation results assess the performance of the proposed policy, in terms of the sum-rate of MTDs and the power budget for different CSI and buffer assumptions.

\section{SySTEM MODEL}

Consider a cellular network which consists of $N$ UEs and a single base station. Underlaid on this network, we consider an M2M system composed of $M$ MTDs, a half-duplex relay, and an MTD gateway for data aggregation. There exist no direct links between the MTDs and the MTD gateway, and data of MTDs is transmitted only via the relay. The considered network architecture mimics a capillary network, in which the basic premise is to keep the M2M data communication autonomous from the cellular network. Here, as is proposed for the emerging cellular networks such as $5 \mathrm{G}$, by using a non- 
orthogonal multiple access (NOMA) procedure, some UEs are transmitting simultaneously in each subcarrier [8]. The total number of subcarriers in the cellular network is $L$. Fig. 1 illustrates the main and interference links in the proposed system model. In this model, time is divided into equal length slots, and all channels experience zero-mean additive white Gaussian noise (AWGN) with unit variance and flat block fading. Therefore, the fading coefficients are constant in each time slot and vary from one time slot to another. In order to store the incoming data from MTDs, the relay is equipped with $M$ infinite-size buffers. However, in Section IV, the effect of limited buffer size on the MTDs' sum-rate will be studied. The amount of the normalized information (bits/symbol) stored in the $j$-th buffer of the relay at the $i$-th time slot is denoted by $Q_{j}(i)$. At the relay, the incoming information from the MTDs is decoded, stored in the related buffer, and finally transmitted to the MTD gateway.

In the $i$-th time slot and $l$-th subcarrier, the channel coefficients between the $j$-th MTD and the relay, between the relay and the MTD gateway, between the $j$-th UE and the relay, and between the $j$-th UE and the MTD gateway are denoted by $h_{j}^{l}(i), h_{M+1}^{l}(i), h_{p 2 j-1}^{l}(i)$, and $h_{p 2 j}^{l}(i)$, respectively. In addition, $g_{j}^{l}(i), g_{M+1}^{l}(i)$, and $g_{p j}^{l}(i)$ represent the channel coefficients between the $j$-th MTD and the base station, between the relay and the base station, and between the $j$-th UE and base station, respectively. We assume that the channel coefficients are stationary and ergodic stochastic processes. The transmit power of the $j$-th UE is denoted by $P_{p j}^{l}(i)$, and furthermore, we let $P_{s j}^{l}(i)$ and $P_{s M+1}^{l}(i)$ denote the transmit power of $j$-th MTD and relay in the $i$-th time slot and $l$ th subcarrier, respectively. $\bar{P}_{s j}$ is the average transmit power of the $j$-th MTD. $\bar{P}_{\text {int }}$ and $P_{\text {int }}^{l}(i)$ represent the average and instantaneous interference power at the base station in the $i$-th time slot and the $l$-th subcarrier.

In this model, instantaneous CSI of the links in the CM2M network is assumed to be available. To this end, the MTD gateway as well as each MTD will transmit pilot signals to the relay and at the relay the channel estimation scheme is applied. In addition, for the interference links from the UEs to the MTDs, I-CSI-I is not known. For CSI of interference links from the MTDs to the UEs, two cases are considered: known I-CSI-I and only the statistical CSI of the interference channels (S-CSI-I) is available. In the proposed protocol, the relay and the MTD gateway treat the interference of the UEs as noise. Assume that for $l$-th subcarrier, $u_{l}$ UEs are transmitting toward the base station concurrently. For the $i$-th time slot and the $l$-th subcarrier, let $C_{j r}^{l}(i)$ and $C_{r d}^{l}(i)$ denote the maximum transmission rates from $j$-th MTD to the relay and from the relay to the MTD gateway, respectively. Hence, $C_{j r}^{l}(i)$ for $j \in\{1, \ldots, M\}$, and $l \in\{1, \ldots, L\}$ and $C_{r d}^{l}(i)$ are obtained as

$$
\begin{aligned}
& C_{j r}^{l}(i)=\log _{2}\left(1+\frac{P_{s j}^{l}(i)\left|h_{j}^{l}(i)\right|^{2}}{1+\sum_{k=1}^{u_{l}} P_{p k}^{l}(i)\left|h_{p 2 k-1}^{l}(i)\right|^{2}}\right), \\
& C_{r d}^{l}(i)=\log _{2}\left(1+\frac{P_{s M+1}^{l}(i)\left|h_{M+1}^{l}(i)\right|^{2}}{1+\sum_{k=1}^{u_{l}} P_{p k}^{l}(i)\left|h_{p 2 k}^{l}(i)\right|^{2}}\right) .
\end{aligned}
$$

Our goal is to select the state of the CM2M network in each time slot and each subcarrier either to be silent or to

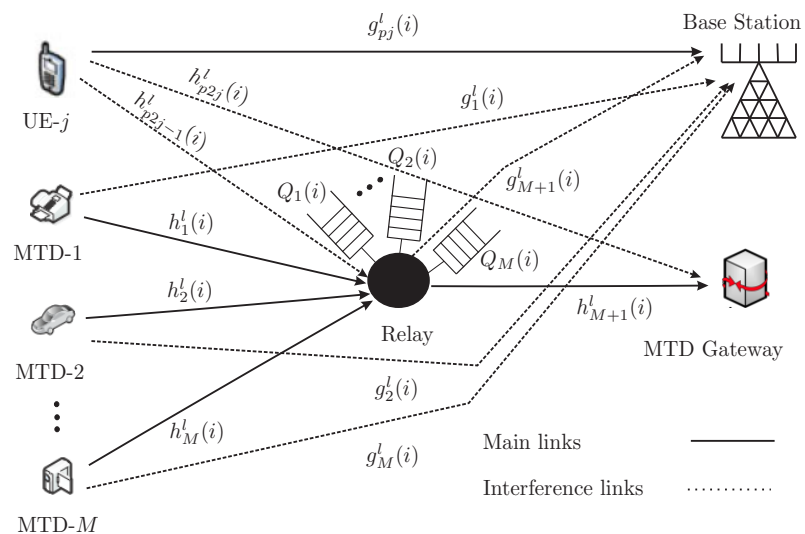

Fig. 1. The considered buffer-aided CM2M communication with multiple MTDs, one relay, and one common MTD gateway.

transmit data. If at a given time slot and subcarrier, CM2M network decides to transmit data, the proposed policy selects either one of the MTDs or the relay for data transmission and allocates its transmit power such that the sum-rate of MTDs is maximized subject to a maximum allowable interference of MTDs at the base station. For this purpose, selection variables $q_{j}^{l}(i) \in\{0,1\}, j \in\{1, \ldots, 2 M\}, l \in\{1, \ldots, L\}$ are defined for the selection of the data transmission state of the MTDs in the $i$-th time slot and the $l$-th subscriber. More specifically, if $q_{j}^{l}(i)=1, j \in\{1, \ldots, M\}, l \in\{1, \ldots, L\}$, the $j$-th MTD is selected for data transmission in the $i$-th time slot and $l$-th subcarrier. In this case, the $j$-th MTD sends data with maximum rate of $R_{j r}^{l}(i)=C_{j r}^{l}(i)$, and at the relay, $R_{j r}^{l}(i)$ normalized information is decoded and stored in the $j$-th buffer. Thus, the amount of information in the queue of $j$-th buffer is increased to $Q_{j}(i)=Q_{j}(i-1)+R_{j r}^{l}(i)$. Otherwise, if $q_{j}^{l}(i)=0$, the $j$-th MTD is not selected in the $i$ th time slot and $l$-th subcarrier. Furthermore, if $q_{j}^{l}(i)=1, j \in$ $\{M+1, \ldots, 2 M\}, l \in\{1, \ldots, L\}$, the relay is selected for data transmission in the $i$-th time slot and $l$-th subcarrier, i.e., the relay reduces $R_{k r d}^{l}(i)=\min \left\{Q_{k}(i-1), C_{r d}^{l}(i)\right\}$ information bits/symbol from $k$-th buffer, where $k=j-M$. Therefore, the amount of normalized information in the queue of $k$-th buffer is decreased to $Q_{k}(i)=Q_{k}(i-1)-R_{k r d}^{l}(i)$. On the other hand, if $q_{j}^{l}(i)=0, j \in\{M+1, \ldots, 2 M\}, l \in\{1, \ldots, L\}$, the relay is silent in time slot $i$ and $l$-th subcarrier. In addition, due to the half-duplex constraint, $\sum_{j=1}^{2 M} q_{j}^{l}(i) \leq 1$ should be hold at each time slot and each subcarrier.

\section{Joint MTD SELEction ANd Power Allocation}

In this section, after studying the achievable average sumrate and the power of the MTDs' interference at the base station, the optimization problem for the MTDs' sum-rate maximization is formulated and solved. In particular, we introduce a joint buffer-aided MTD selection and power allocation protocol as a solution of this problem.

\section{A. Achievable Average Sum-Rate}

In this paper, we assume that MTDs always have data to transmit and the number of time slots, $N$, tends to infinity. Hence, the average transmission rates of the $j$-th MTD to the relay, the $j$-th buffer of relay to the MTD gateway, and the relay to the MTD gateway, denoted by $\bar{R}_{j r}, \bar{R}_{j r d}$, and $\bar{R}_{r d}$, respectively, are as follows

$$
\bar{R}_{j r}=\lim _{N \rightarrow \infty} \frac{1}{L N} \sum_{l=1}^{L} \sum_{i=1}^{N} q_{j}^{l}(i) C_{j r}^{l}(i),
$$




$$
\begin{array}{r}
\bar{R}_{j r d}=\lim _{N \rightarrow \infty} \frac{1}{L N} \sum_{l=1}^{L} \sum_{i=1}^{N} q_{j+M}^{l}(i) \min \left\{C_{r d}^{l}(i), Q_{j}(i-1)\right\},(4) \\
\bar{R}_{r d}=\frac{1}{L} \sum_{l=1}^{L} \sum_{j=1}^{M} \bar{R}_{j r d}^{l}=\lim _{N \rightarrow \infty} \frac{1}{L N} \sum_{l=1}^{L} \sum_{i=1}^{N}(5) \\
\sum_{j=1}^{M} q_{j+M}^{l}(i) \min \left\{C_{r d}^{l}(i), Q_{j}(i-1)\right\} .
\end{array}
$$

Assuming availability of enough information in the buffers, the average processing rate of the $j$-th buffer of the relay is

$$
\bar{R}_{j r d}=\lim _{N \rightarrow \infty} \frac{1}{L N} \sum_{l=1}^{L} \sum_{i=1}^{N} q_{j+M}^{l}(i) C_{r d}^{l}(i) .
$$

For rate stability of an infinite-size buffer, the average input rate should be equal to the average processing rate, i.e., $\bar{R}_{j r}=$ $\bar{R}_{j r d}^{*}$ [9]. Therefore, $\bar{R}_{j r d}=\bar{R}_{j r d}^{*}$ is also valid, see [10] for a proof. The instantaneous interference (I-INT) power of MTDs at the base station can be written as

$$
\begin{aligned}
& P_{\text {int }}^{l}(i)=\sum_{j=1}^{M} q_{j}^{l}(i) P_{s j}^{l}(i)\left|g_{j}^{l}(i)\right|^{2}+ \text { (7) } \\
& \sum_{j=M+1}^{2 M} q_{j}^{l}(i) P_{s M+1}^{l}(i)\left|g_{M+1}^{l}(i)\right|^{2},
\end{aligned}
$$

The average interference (A-INT) power of MTDs for I-CSI-I and S-CSI-I assumptions are

$$
\bar{P}_{\mathrm{int}}=\left\{\begin{array}{c}
\frac{1}{L N} \sum_{l=1}^{L} \sum_{i=1}^{N}\left(\sum_{j=1}^{M} q_{j}^{l}(i) P_{s j}^{l}(i)\left|g_{j}^{l}(i)\right|^{2}\right. \\
\left.+\sum_{j=M+1}^{2 M} q_{j}^{l}(i) P_{s M+1}^{l}(i)\left|g_{M+1}^{l}(i)\right|^{2}\right), \text { I-CSI-I }, \\
\frac{1}{L N} \sum_{l=1}^{L} \sum_{i=1}^{N}\left(\sum_{j=1}^{M} \Omega_{j}^{l} q_{j}^{l}(i) P_{s j}^{l}(i)\right. \\
\left.+\Omega_{M+1}^{l} \sum_{j=M+1}^{2 M} q_{j}^{l}(i) P_{s M+1}^{l}(i)\right), \quad \text { S-CSI-I }
\end{array}\right.
$$

in which $\Omega_{j}^{l}=\mathbb{E}\left\{\left|g_{j}^{l}(i)\right|^{2}\right\}, j \in\{1, \ldots, M+1\}$, and $\mathbb{E}\{\cdot\}$ denotes expectation.

\section{B. Optimal MTD Selection Protocol}

The sum-rate of MTDs can be maximized by solving the below optimization problem

$$
\begin{array}{cl}
\underset{q_{j}^{l}(i), P_{s k}^{l}(i), \forall i, j, k, l}{\operatorname{maximize}} & \bar{R}_{r d}^{*} \\
\text { subject to C1: } & \bar{P}_{\text {int }} \leq I_{t h r} \quad \text { or } \quad P_{\text {int }}^{l}(i) \leq I_{t h r} \\
\text { C2: } & \bar{R}_{j r}=\bar{R}_{j r d}^{*}, \forall j \in\{1, \ldots, M\} \\
\text { C3: } & \boldsymbol{q}^{l}(i) \in \mathcal{Q}, \forall i, \\
\text { C4: } & P_{s k}^{l}(i) \geq 0, \forall k \in\{1, \ldots, M+1\} \\
\text { C5: } & \sum_{k=1}^{M+1} \bar{P}_{s k} \leq P_{t},
\end{array}
$$

in which $\boldsymbol{q}^{l}(i)=\left[q_{1}^{l}(i), \ldots, q_{2 M}^{l}(i)\right]$. Constraint $\mathrm{C} 1$ limits the A-INT power of MTDs and the I-INT power of MTDs at the base station to be below a predefined threshold $I_{t h r}$. Rate stability of the buffers at the relay are ensured by constraints C2. The set $\mathcal{Q}$ is defined as $\mathcal{Q}=\left\{\left[q_{1}^{l}(i), \ldots, q_{2 M}^{l}(i)\right] \mid q_{j}^{l}(i) \in\right.$ $\left.\{0,1\} \wedge \sum_{j=1}^{2 M} q_{j}^{l}(i) \leq 1\right\}$. Constraint $\mathrm{C} 4$ ensures that the MTD's transmit powers have positive values and constraint $\mathrm{C} 5$ limits the sum of the MTDs' average transmit powers to be under a predefined value, $P_{t}$.

Theorem 1. For both I-CSI-I and S-CSI-I cases and both the A-INT and I-INT power conditions, the optimal selection variables that maximize the MTDs' sum-rate are obtained as follows

$$
\boldsymbol{q}^{l}(i)= \begin{cases}{[0,0, \ldots, 0,0],} & \text { if } \Gamma_{j}^{l}(i)<0, \text { for } 1 \leq j \leq 2 M \\ {[1,0, \ldots, 0,0],} & \text { if } \Gamma_{1}^{l}(i) \geq \Gamma_{j}^{l}(i) \geq 0 \\ & \text { OR } \Gamma_{1}^{l}(i)>0>\Gamma_{j}^{l}(i), \\ & \text { for } 2 \leq j \leq 2 M, \\ \vdots & \\ {[0,0, \ldots, 0,1],} & \text { if } \Gamma_{2 M}^{l}(i)>\Gamma_{j}^{l}(i)>0 \\ & \text { OR } \Gamma_{2 M}^{l}(i)>0>\Gamma_{j}^{l}(i), \\ & \text { for } 1 \leq j \leq 2 M-1,\end{cases}
$$

in which $\Gamma_{j}^{l}(i)$ for $1 \leq j \leq M$ and $M+1 \leq j \leq 2 M^{(10)}$ are given in (11) and (12), respectively.

$$
\begin{aligned}
& \left\{\begin{array}{c}
\lambda_{j} C_{j r}^{l}(i)-\mu P_{s j}^{l}(i)\left|g_{j}^{l}(i)\right|^{2} \\
-\gamma P_{s j}^{l}(i), \\
\hat{\lambda}_{j} C_{j r}(i)-\hat{\mu} P_{s j}^{l}(i) \Omega_{j}^{l}
\end{array} \quad\right. \text { A-INT, I-CSI-I, }
\end{aligned}
$$

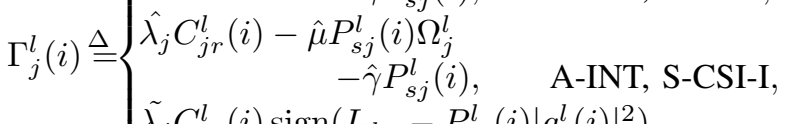

$$
\begin{aligned}
& \begin{array}{r}
\tilde{\lambda}_{j} C_{j r}^{l}(i) \operatorname{sign}\left(I_{t h r}-P_{s j}^{l}(i)\left|g_{j}^{l}(i)\right|^{2}\right) \\
-\tilde{\gamma} P_{s j}^{l}(i), \quad \text { I-INT, }
\end{array}
\end{aligned}
$$

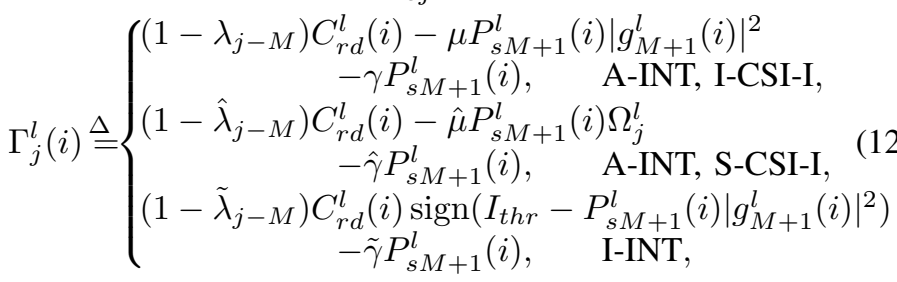$$
\text { Proof: Please refer to Appendix A. }
$$

Theorem 2. The transmit power of the MTDs in the $i$-th time slot and $l$-th subcarrier, i.e., $P_{s k}^{l}(i), 1 \leq k \leq M, 1 \leq l \leq L$ is given by

$$
P_{s k}^{l}(i)=\left\{\begin{array}{cl}
\frac{\lambda_{k}}{\ln (2)\left(\gamma+\mu\left|g_{k}^{l}(i)\right|^{2}\right)}+ & \\
-\frac{\sum_{j=1}^{u} P_{p j}^{l}(i)\left|h_{p 2 j-1}^{l}(i)\right|^{2}}{\left|h_{k}^{l}(i)\right|^{2}}, & \text { A-INT, I-CSI-I, } \\
\frac{\hat{\lambda}_{k}}{\ln (2)\left(\hat{\gamma}+\hat{\mu} \Omega_{l}^{l}\right)}+ & \\
-\frac{\left.\sum_{j=1}^{u_{l}} P_{p j}^{l}(i)\right|^{2} \mathbb{E}\left\{\mid h_{p 2 j-1}^{l}(i)\right\}}{\left|h_{k}^{l}(i)\right|^{2}}, & \text { A-INT, S-CSI-I, } \\
\frac{\tilde{\lambda}_{k}}{\ln (2) \tilde{\gamma}}-\frac{\sum_{j=1}^{u_{l}} P_{p j}^{l}(i)\left|h_{p 2 j-1}^{l}(i)\right|^{2}}{\left|h_{k}^{l}(i)\right|^{2}}, & \text { I-INT. }
\end{array}\right.
$$

Similarly, the transmit power of the relay in the $i$-th time slot and $l$-th subcarrier, i.e., $P_{s M+1}^{l}(i)$ is given by

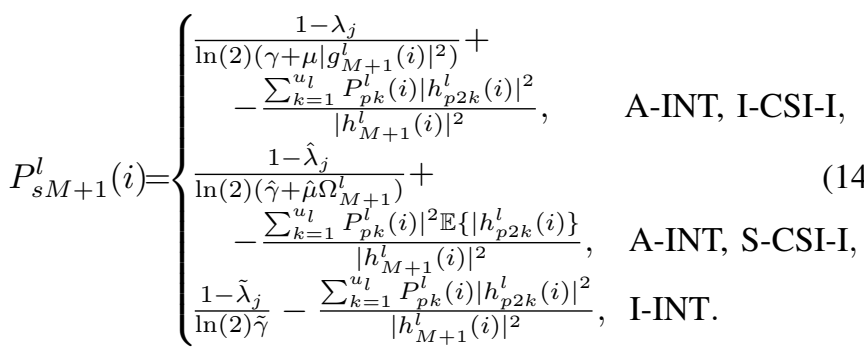

where $j$ is in the interval of $1 \leq j \leq M$ such that $g_{M+j}^{l}=1$ holds.

Proof: Please refer to Appendix B.

For A-INT case with I-CSI-I and S-CSI-I knowledge, constants $\left(\mu, \lambda_{1}, \ldots, \lambda_{M}, \gamma\right)$ and $\left(\hat{\mu}, \hat{\lambda}_{1}, \ldots, \hat{\lambda}_{M}, \hat{\gamma}\right)$ can be obtained by a $M+2$-dimensional search such that conditions $\mathrm{C} 1, \mathrm{C} 2$, and C5 in (9) are satisfied. Similarly, for I-INT case, constants $\left(\tilde{\lambda}_{1}, \ldots, \tilde{\lambda}_{M}, \tilde{\gamma}\right)$ are achieved by a $M+1$-dimensional search such that conditions C2 and C5 in (9) are met. By using 


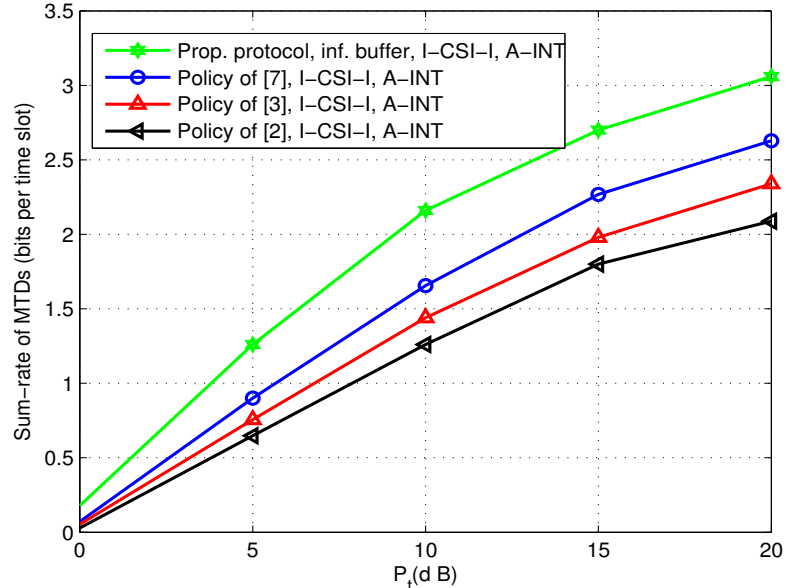

Fig. 2. MTDs' sum-rate versus the MTDs' power budget, $P_{t}(\mathrm{~dB})$, for five MTDs.

the proposed buffer-aided MTD selection scheme in (10), in each time slot and each subcarrier, CM2M network decides optimally to be silent or to transmit data. If CM2M network decides to transmit data, a transmitter node with maximum transmission rate (either one of the MTDs or the relay) is selected for data transmission. Therefore, the sum-rate of MTDs is maximized while the induced interference of MTDs to the base station is limited.

\section{Simulation Results and Analysis}

In this section, we assess the proposed policy in terms of the sum-rate MTDs and the MTDs' power budget through simulations. There exist five MTDs in the CM2M network and we assume Rayleigh block fading. We assume that $P_{t}=4$ $\mathrm{dB}, P_{p}^{l}(i)=14 \mathrm{~dB}, \mathbb{E}\left\{\left|g_{p}^{l}(i)\right|^{2}\right\}=0.1, \mathbb{E}\left\{\left|h_{j}^{l}(i)\right|^{2}\right\}=1$, and $\mathbb{E}\left\{\left|h_{p 1}^{l}(i)\right|^{2}\right\}=\mathbb{E}\left\{\left|h_{p 2}^{l}(i)\right|^{2}\right\}=\Omega_{j}^{l}=0.1$, i.e., the CM2M network has smaller size in comparison with the cellular network. The sum-rate of MTDs is illustrated versus the interference threshold, $I_{t h r}$, in Fig. 2 for S-CSI-I and ICSI-I cases, A-INT and I-INT power conditions, and infinite and limited buffer size. For investigating the limited buffer size effect, we assume that the size of the buffer equals to ten times of the maximum MTDs' sum-rate obtained with the proposed policy for the infinite buffer size.

Fig. 2 illustrates the MTDs' sum-rate of our proposed protocol, the proposed policies in [2], [3], and [7] versus the MTDs' power budget, $P_{t}$, for a CM2M with five MTDs. As this figure shows, by increasing the MTDs' power budget, the MTDs' sum-rate is enhanced. Furthermore, due to the MTDs' power allocation, our proposed policy outperforms other schemes in terms of the MTDs' sum-rate.

In Fig. 3, the MTDs' sum-rate of our introduced scheme, the proposed protocols in [2], [3], and [7] are shown versus the number of MTDs, $M$. As can be seen from Fig. 3, increasing the number of MTDs results in enhancement of the degrees of freedom which causes improvement of the MTDs' sum-rate. In Fig. 2 and 3, the proposed buffer-aided MTD selection policy with the A-INT power condition, infinite buffer size, and ICSI-I knowledge has the largest MTDs' sum-rate among all the other schemes.

\section{APPENDIX A}

We first solve the optimization problem in (9) for the case of A-INT with I-CSI-I knowledge. The optimization problem

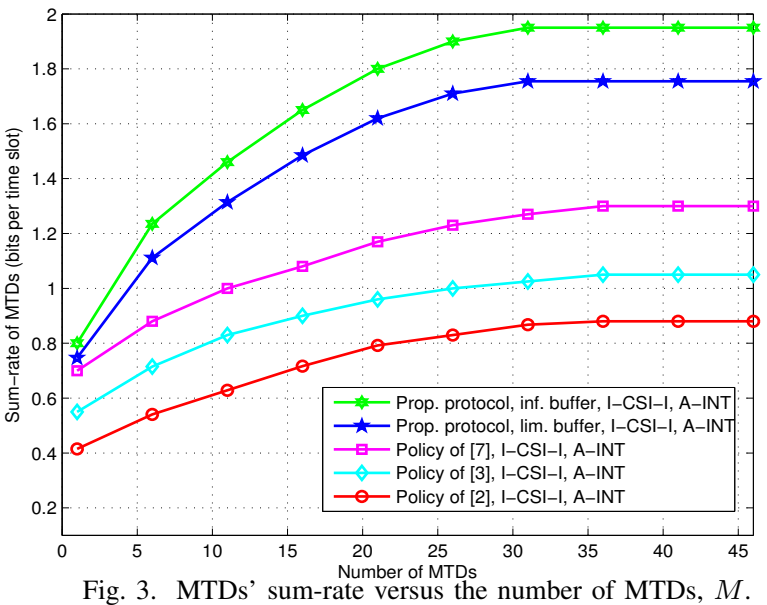

in (9) is an integer program which is classified as a nondeterministic polynomial-time hard problem. Thus, the binary constraint $q_{j}^{l}(i) \in\{0,1\}, j \in\{1, \ldots, 2 M\}, l \in\{1, \ldots, L\}$ in (9) is relaxed to condition $0 \leq q_{j}^{l}(i) \leq 1$. Nevertheless, because the relaxed problem is a linear programming problem in terms of $q_{j}^{l}(i)$, the optimal solution can be obtained at the intersection of the feasible hyperplanes, i.e., $q_{j}^{l}(i)=0$ or $q_{j}^{l}(i)=1$. Therefore, the optimal solution of the optimization problem is not influenced by the relaxation. For the relaxed linear program, the Karush-Kuhn-Tucker (KKT) conditions are both necessary and sufficient conditions, and therefore, the optimal solution can be achieved from the KKT conditions. In the following, the KKT conditions for the relaxed optimization problem are studied.

The Lagrangian function for the relaxed optimization problem is provided as $\mathcal{L}\left(q_{j}^{l}(i), P_{k}^{l}(i), \mu, \lambda_{1}, \ldots, \lambda_{M}, \alpha_{j}^{l}(i), \beta_{j}^{l}(i), \varphi^{l}(i), \nu_{k}^{l}(i), \gamma\right)=$ $-\sum_{j=1}^{M} \bar{R}_{j r d}^{*}+\mu\left(\bar{P}_{\text {int }}-I_{t h r}\right)+\sum_{j=1}^{M} \lambda_{j}\left(\bar{R}_{j r d}^{*}-\bar{R}_{j r}\right)$ $+\gamma\left(\sum_{k=1}^{M+1} \bar{P}_{s k}-P_{t}\right)+\sum_{l=1}^{L} \sum_{i=1}^{N} \sum_{j=1}^{2 M} \alpha_{j}^{l}(i)\left(q_{j}^{l}(i)-1\right)$ $-\sum_{l=1}^{L} \sum_{i=1}^{N} \sum_{j=1}^{2 M} \beta_{j}^{l}(i) q_{j}^{l}(i)+\sum_{l=1}^{L} \sum_{i=1}^{N} \varphi^{l}(i) \times$ $\left(\sum_{j=1}^{2 M} q_{j}^{l}(i)-1\right)-\sum_{l=1}^{L} \sum_{i=1}^{N} \sum_{k=1}^{M+1} P_{s k}^{l}(i) \nu_{k}^{l}(i)$,

in which $\mu, \lambda_{j}, \alpha_{j}^{l}(i), \beta_{j}^{l}(i), \varphi^{l}(i), \nu_{k}^{l}(i)$, and $\gamma$ are the Lagrangian multipliers for constraints $\mathrm{C} 1, \mathrm{C} 2, q_{j}^{l}(i) \leq 1$, $q_{j}^{l}(i) \geq 0, \sum_{j=1}^{2 M} q_{j}^{l}(i) \leq 1, \mathrm{C} 4$, and $\mathrm{C} 5$ respectively. In the following, the derivations of Lagrangian function in (15) with respect to $q_{j}^{l}(i)$ for $1 \leq j \leq M$, and $M+1 \leq j \leq 2 M$ are equated to zero in (16) and (17), respectively.

$$
\begin{gathered}
\left.\frac{\partial \mathcal{L}}{\partial q_{j}^{l}(i)}\right|_{1 \leq j \leq M}=\frac{\mu}{N} P_{s j}^{l}\left|g_{j}^{l}(i)\right|^{2}-\frac{\lambda_{j}}{N} C_{j r}^{l}(i)+\frac{\gamma}{N} P_{s j}^{l}(i)(16) \\
+\alpha_{j}^{l}(i)-\beta_{j}^{l}(i)+\varphi^{l}(i)=0 \\
\left.\frac{\partial \mathcal{L}}{\partial q_{j}^{l}(i)}\right|_{M+1 \leq j \leq 2 M}=\frac{-C_{r d}^{l}(i)}{N}+\frac{\mu}{N} P_{s M+1}^{l}(i)\left|g_{M+1}^{l}(i)\right|^{2}(17) \\
+\frac{\lambda_{j}}{N} C_{r d}^{l}(i)+\frac{\gamma}{N} P_{s M+1}^{l}(i)+\alpha_{j}^{l}(i)-\beta_{j}^{l}(i)+\varphi^{l}(i)=0 .
\end{gathered}
$$

If $q_{1}^{l}(i)=1$ holds, other selection variables are zero, i.e., $q_{j}^{l}(i)=0$ for $2 \leq j \leq 2 M$, and based on the complementary slackness constraint, $\alpha_{j}^{l}(i)$ for $2 \leq j \leq 2 M$ and $\beta_{1}^{l}(i)$ are 
TABLE I

NECESSARY CONDITIONS FOR OPTIMAL $\boldsymbol{q}^{l}(i)$

\begin{tabular}{|c|c|}
\hline $\boldsymbol{q}^{l}(i)$ & Necessary conditions \\
\hline$[1,0, \ldots, 0,0]$ & $\Gamma_{1}^{l}(i) \geq 0, \Gamma_{1}^{l}(i) \geq \Gamma_{j}^{l}(i)$, for $1 \leq j \leq 2 M$. \\
\hline$[0,1,0, \ldots, 0]$ & $\Gamma_{2}^{l}(i) \geq 0, \Gamma_{2}^{l}(i) \geq \Gamma_{j}^{l}(i)$ for $1 \leq j \leq 2 M$. \\
\hline$\vdots$ & $\vdots$ \\
\hline$[0,0, \ldots, 0,1]$ & $\Gamma_{2 M}^{l}(i) \geq 0, \Gamma_{2 M}^{l}(i) \geq \Gamma_{j}^{l}(i)$, for $1 \leq j \leq 2 M$. \\
\hline$[0,0, \ldots, 0,0]$ & $\Gamma_{j}^{l}(i) \leq 0$, for $1 \leq j \leq 2 M$. \\
\hline
\end{tabular}

zero [11]. By substituting these variables into (16) and (17), we have

$$
\begin{aligned}
N\left(\varphi^{l}(i)+\alpha_{1}^{l}(i)\right) & =\lambda_{1} C_{1 r}^{l}(i)-\mu P_{s 1}^{l}(i)\left|g_{1}^{l}(i)\right|^{2} \\
& -\gamma P_{s 1}^{l}(i) \triangleq \Gamma_{1}^{l}(i),
\end{aligned}
$$

and for $2 \leq j \leq M$, we obtain

$$
\begin{aligned}
N\left(\varphi^{l}(i)-\beta_{j}^{l}(i)\right) & =\lambda_{j} C_{j r}^{l}(i)-\mu P_{s j}^{l}(i)\left|g_{j}^{l}(i)\right|^{2} \\
& -\gamma P_{s j}^{l}(i) \triangleq \Gamma_{j}^{l}(i),
\end{aligned}
$$

and for $M+1 \leq j \leq 2 M$, we obtain

$$
\begin{aligned}
& N\left(\varphi^{l}(i)-\beta_{j}^{l}(i)\right)=\left(1-\lambda_{j-M}\right) C_{r d}^{l}(i)+ \\
& -\mu P_{s M+1}^{l}(i)\left|g_{M+1}^{l}(i)\right|^{2}-\gamma P_{s M+1}^{l}(i) \triangleq \Gamma_{j}^{l}(i),
\end{aligned}
$$

where $\Gamma_{j}^{l}(i)$ is the selection metric in the $i$-th time slot and $l$-th subcarrier. Based on the dual feasibility constraint, $\beta_{j}^{l}(i), \varphi^{l}(i), \alpha_{j}^{l}(i) \geq 0$. Hence, $\Gamma_{1}^{l}(i)$ in (18) is non-negative. In addition, by subtracting the expression in (18) from (19) and the expression in (18) from (20), we have

$$
\Gamma_{1}^{l}(i)-\Gamma_{j}^{l}(i)=N\left(\alpha_{1}^{l}(i)+\beta_{j}^{l}(i)\right) \geq 0 .
$$

Therefore, $\Gamma_{1}^{l}(i) \geq 0$ and $\Gamma_{1}^{l}(i) \geq \Gamma_{j}^{l}(i), 2 \leq j \leq 2 M$ are the necessary conditions for $\boldsymbol{q}^{l}(i)=[1,0, \ldots, 0]$. Similarly, the other necessary conditions are achieved in Table-I.

Since the probability of equality of two selection metrics is zero, i.e., $\operatorname{Pr}\left\{\Gamma_{j}^{l}(i)=\Gamma_{k}^{l}(i)\right\}=0$, when $\Gamma_{j}^{l}(i)$ and $\Gamma_{k}^{l}(i)$ are equal, selecting either $q_{j}^{l}(i)=1$ or $q_{k}^{l}(i)=1$ does not alter the maximum sum-rate of MTDs. Hence, the necessary conditions in Table-I are also sufficient which results in Theorem 1. The Lagrangian multipliers $\mu, \lambda_{j}$, and $\gamma$ rely on the statistics of the channels and can be determined by a $M+2$-dimensional search such that the conditions C1, C2, and C5 in (9) are satisfied. In (15), $\mu$ and $\gamma$ are the Lagrangian multipliers of the inequality constraints, and therefore, $\mu \geq 0$ and $\gamma \geq 0$ are valid. In addition, if $\Gamma_{j}^{l}(i) \leq 0$ and $\lambda_{j}<0$ hold, the $j$-th MTD is not selected for the data transmission which results in zero throughput for $j$-th MTD, and hence, $\Gamma_{j}^{l}(i) \leq 0$ and $\lambda_{j}<0$ are not the case. In addition, if $\lambda_{j}>1$ hold the $j$-th buffer of the relay is not selected to transmit the data to the MTD gateway, and therefore, $0 \leq \lambda_{j} \leq 1$ should be held.

For S-CSI-I knowledge, the optimization problem can be solved similar to the I-CSI-I case by replacing $\Omega_{j}^{l}$ by $\left|g_{j}^{l}(i)\right|^{2}$. For I-INT power condition, the maximization problem can be solved in a similar way as in [10] with the difference that the I-INT power condition should be met in each time slot. Therefore, for the I-INT power constraint, $\Gamma_{j}^{l}(i)$ should be multiplied with $\operatorname{sign}\left(I_{t h r}-P_{s j}^{l}(i)\left|g_{j}^{l}(i)\right|^{2}\right)$ in the proposed MTD selection scheme. This completes the proof.

\section{APPENDIX B}

In order to calculate the optimal transmit power of MTDs and relay, we differentiate the Lagrangian function in (15) with respect to $P_{s k}^{l}(i)$, for $1 \leq k \leq M+1$, and equate them to zero. Therefore, for $1 \leq k \leq M$, we have

$$
\begin{aligned}
& \frac{\partial \mathcal{L}}{\partial P_{s k}^{l}(i)}=\frac{\gamma}{N} q_{k}^{l}(i)+\frac{\bar{\mu}}{N} q_{k}^{l}(i)\left|g_{k}^{l}(i)\right|^{2}-\nu_{k}^{l}(i)+ \\
& -\frac{\lambda_{k}}{N} \frac{q_{k}^{l}(i)\left|h_{k}^{l}(i)\right|^{2}}{\ln (2)\left(1+\sum_{j=1}^{u_{l}} P_{p j}^{l}(i)\left|h_{p 2 j-1}^{l}(i)\right|^{2}+P_{s k}^{l}(i)\left|h_{k}^{l}(i)\right|^{2}\right)}=0,
\end{aligned}
$$

and

$$
\begin{aligned}
& \frac{\partial \mathcal{L}}{\partial P_{s M+1}^{l}(i)}=\frac{\gamma}{N} \sum_{j=M+1}^{2 M} q_{j}^{l}(i)+\frac{\mu}{N} \sum_{j=M+1}^{2 M} q_{j}^{l}(i)\left|g_{M+1}^{l}(i)\right|^{2} \\
& +\frac{\left|h_{M+1}^{l}(i)\right|^{2}\left(\sum_{j=1}^{M} \lambda_{j} q_{M+j}^{l}(i)-\sum_{j=M+1}^{2 M} q_{j}^{l}(i)\right)}{N \ln (2)\left(1+\theta+P_{s M+1}^{l}(i)\left|h_{M+1}^{l}(i)\right|^{2}\right)} \\
& -\nu_{M+1}^{l}(i)=0,
\end{aligned}
$$

in which $\theta=\sum_{k=1}^{u_{l}} P_{p k}^{l}(i)\left|h_{p 2 k}^{l}(i)\right|^{2}$. Assume that at the $i$ th time slot and $l$-th subcarrier, the $k$-th MTD is selected to transmit its data to the relay, and thus, $q_{k}^{l}(i)=1, P_{s k}^{l}(i) \neq 0$. According to the complementary slackness condition, $\nu_{k}^{l}(i)=$ 0 holds. Therefore, by substituting $q_{k}^{l}(i), \nu_{k}^{l}(i)$ into (22), for $1 \leq k \leq M$ we obtain

$$
P_{s k}^{l}(i)=\frac{\lambda_{k}}{\ln (2)\left(\gamma+\mu\left|g_{k}^{l}(i)\right|^{2}\right)}-\frac{\sum_{j=1}^{u_{l}} P_{p j}^{l}(i)\left|h_{p 2 j-1}^{l}(i)\right|^{2}}{\left|h_{k}^{l}(i)\right|^{2}}
$$

Moreover, if at the $i$-th time slot and $l$-th subcarrier, the relay is selected to transmit the MTDs' data from the $k$-th buffer to the MTD gateway, $q_{k+M}^{l}(i)=1, P_{s M+1}^{l}(i) \neq 0$, and hence, based on complementary slackness constraint, we have $\nu_{M+1}^{l}(i)=$ 0 . Thus, by substituting $q_{k+M}^{l}(i)$ and $\nu_{M+1}^{l}(i)$ into (23), for $1 \leq k \leq M, q_{k+M}(i)=1$, we have

$$
\begin{aligned}
& P_{s M+1}^{l}(i)=\frac{1-\lambda_{j}}{\ln (2)\left(\gamma+\mu\left|g_{M+1}^{l}(i)\right|^{2}\right)}+ \\
& \text { This completes the proof. }
\end{aligned}
$$

\section{REFERENCES}

[1] I. B. S. Group, Internet of Everything, Cisco, Tech. Rep.

[2] Y. Li, K. K. Chai, Y. Chen, and J. Loo, "Duty Cycle Control With Joint Optimisation of Delay and Energy Efficiency for Capillary Machine-toMachine Networks in 5G Communication System", Trans. Emerging Tel. Tech., pp. 56-69, DOI:10.1002/ett.2891, 2015.

[3] Sh. Lin, and K. Chen, "Cognitive and Opportunistic Relay for QoS Guarantees in Machine-to-Machine Communications," IEEE Trans. Mobile Computing, doi: 10.1109/TMC.2015.2421931, 2015.

[4] R. Boisguene, S. Chou, C. Huang, "A Survey on Cognitive Machine-toMachine Communications," in Proc. IEEE ICWCMC, pp. 739-744, Aug. 2014.

[5] Y. Zhang, R. Yu, M. Nekovee, Y. Liu, S. Xie, and S. Gjessing, "Cognitive Machine-to-Machine Communications: Visions and Potentials for the Smart Grid", IEEE Network pp. 6-13, 2013.

[6] F. Bader, P. Demestricas, L. DaSilva, and H. Harada, "Cognitive Radio in Emerging Communications Systems Small Cells, Machine-to-Machine Communications, TV White Spaces and Green Radios", Trans. Emerging Tel. Tech., PP. 633-635, 2013.

[7] D. Tarchi, R. Fantacci, and D. Marabissi, "An M2M Cognitive MAC Protocol for Overlaid OFDMA Environments", Trans. Emerging Tel. Tech., doi: 10.1002/ett.2955, 2015.

[8] M. Al-Imari, X. Pei, M. A. Imran, and R. Tafazolli, "Uplink NonOrthogonal Multiple Access for 5G Wireless Networks," in Proc. IEEE ISWCS, pp.781-785, Aug. 2014.

[9] M. J. Neely, Stochastic Network Optimization with Application to Communication and Queueing Systems. Morgan \& Claypool, 2010.

[10] N. Zlatanov, R. Schober, and P. Popovski, "Buffer-Aided Relaying with Adaptive Link Selection," IEEE J. Sel. Areas Commun., vol. 31, no. 8, pp. 1530-1542, Aug. 2013.

[11] S. Boyd and L. Vandenberghe, Convex Optimization. Cambridge Univ. press, 2009. 